\title{
Estudio seroepidemiológico de Toxoplasma gondii en caprinos del Perú y su asociación a factores de riesgo
}

\author{
Seroepidemiological study of Toxoplasma gondii in Peruvian goats and its \\ association with risk factors
}

\author{
Amanda Chávez V. ${ }^{1,5}$, Rosa Pinedo V. ${ }^{1}$, Francisco Suárez A. ${ }^{2}$, Eglinton Villacaqui A. ${ }^{3}$, \\ Ibelice Pérez $\mathrm{C}^{4}$
}

\section{Resumen}

\begin{abstract}
El objetivo del estudio fue determinar la seroprevalencia y su asociación con los factores de riesgo en la infección por Toxoplasma gondii en caprinos del Perú. Se trabajó con un grupo de 1119 sueros de caprinos colectados entre 2017-2018 de 23 de los 24 departamentos del país por el Servicio Nacional de Sanidad Animal (SENASA) para un monitoreo de Brucella melitensis. El número de muestras fue proporcional a la población de cabras de cada departamento. Se consideraron las variables edad $(<1,1-3,>3$ años), sexo, procedencia (Zona Costa Norte, Zona Costa Centro-Sur, Zona Sierra-oriente, altitud (0-500, >500-2500, >2500 msnm) y tipo de crianza (intensiva, extensiva). Los sueros fueron analizados con un kit comercial de ELISA indirecta multi-especie y los factores de riesgo se determinaron mediante regresión logística. La prevalencia general
\end{abstract}

\footnotetext{
${ }^{1}$ Laboratorio de Microbiología y Parasitología Veterinaria, Facultad de Medicina Veterinaria, Universidad Nacional Mayor de San Marcos, Lima, Perú

${ }^{2}$ Laboratorio de Epidemiología y Economía Veterinaria, Facultad de Medicina Veterinaria, Universidad Nacional Mayor de San Marcos, Lima, Perú

${ }^{3}$ Laboratorio de Epidemiología Veterinaria y Salud Pública, Facultad de Medicina Veterinaria y Zootecnia, Universidad Cientifica del Sur, Lima, Perú

4. Servicio Nacional de Sanidad Agraria del Perú - SENASA, Lima, Perú

${ }^{5}$ E-mail: achavezv@unmsm.edu.pe
}

Recibido: 5 de abril de 2020

Aceptado para publicación: 24 de noviembre de 2021

Publicado: 22 de diciembre de 2021

CLos autores. Este artículo es publicado por la Rev Inv Vet Perú de la Facultad de Medicina Veterinaria, Universidad Nacional Mayor de San Marcos. Este es un artículo de acceso abierto, distribuido bajo los términos de la licencia Creative Commons Atribución 4.0 Internacional (CC BY 4.0) [https:// creativecommons.org/licenses/by/4.0/deed.es] que permite el uso, distribución y reproducción en cualquier medio, siempre que la obra original sea debidamente citada de su fuente original 
encontrada de $T$. gondii fue de $28.15 \%$ (IC 95\%: 25.5-30.9). La mayor prevalencia se encontró en la Sierra-oriente y Costa Centro-Sur), a una altitud $<2500$ msnm y bajo crianza intensiva.

Palabras clave: cabra, ELISA, toxoplasmosis, crianza intensiva, zoonosis parasitaria

\section{Abstract}

The aim of this study was to determine the seroprevalence of Toxoplasma gondii and its association with risk factors in Peruvian goats. A total of 1119 goat sera collected between 2017-2018 from 23 of the 24 departments of the country by the National Animal Health Service (SENASA) to monitor Brucella melitensis were evaluated. The number of samples was proportional to the goat population of each department. The variables age $(<1,1-3,>3$ years), sex, origin (North Coast Zone, Central-South Coast Zone, Sierra-East Zone, altitude (0-500, $>500-2500,>2500 \mathrm{~m})$ and type of rearing (intensive, extensive) were considered. The sera were analysed with a commercial multi-species indirect ELISA kit and the risk factors were determined by logistic regression. The overall prevalence of $T$. gondii was $28.15 \%$ (95\% CI: 25.5-30.9). The highest prevalences were found in the SierraEast and Central-South Coast), at an altitude $<2500 \mathrm{~m}$ and under intensive rearing.

Key words: goat, ELISA, toxoplasmosis, intensive rearing, parasitic zoonosis

\section{INTRODUCCIÓN}

La toxoplasmosis, causada por Toxoplasma gondii, constituye una de las enfermedades zoonóticas más generalizada a nivel mundial (Tenter et al., 2000). Presenta como hospedero definitivo a los felinos domésticos y silvestres y como hospederos intermediarios lo constituyen una amplia gama de animales de sangre caliente (Dubey y Jones, 2008). Se estima que aproximadamente el $22 \%$ de la infección humana por Toxoplasma se debe al consumo de carne insuficientemente cocida (FAO-WHO, 2015).

El ciclo de vida de $T$. gondii presenta tres formas infectivas: el ooquiste que alberga esporozoitos, el cual es eliminado al medio ambiente junto con las heces de los felinos; los quistes tisulares conteniendo bradizoitos, formas de multiplicación lenta que se encuentran en músculo, órganos y el sistema nervioso central (Dubey y Jones, 2008). Además los taquizoitos son formas de multiplicación rápida, que intervienen en la transmisión congénita, la cual ocurre en el hombre, oveja y cabra (Dubey y Jones, 2008). El hombre y animales de sangre caliente se pueden infectar por primera vez al consumir alimentos y agua contaminados con ooquistes infectivos o al ingerir carne poco cocida conteniendo quistes tisulares.

La toxoplasmosis en humanos puede causar enfermedades graves como encefalitis toxoplasmática en pacientes inmunodeprimidos y abortos o causar defectos congénitos en fetos (Montoya y Liesenfeld, 2004). La ingestión de carne de cordero infectado poco cocido es un factor de riesgo importante para la infección por $T$. gondii en mujeres embarazadas, personas inmunodeprimidas y seronegativas a este parásito (Cook et al., 2000). Se estima que el $50 \%$ de casos registrados ocurre por esta vía (Scallan et al., 2011). Un estudio reciente reveló una distribución desigual de $T$. gondii en la musculatura en cabras y que incluso pequeñas porciones de carne (5-10 g) tienen el potencial 
de transmitir $T$. gondii si se consumen crudas o poco cocidas (Rani et al., 2020).

Del punto de vista de sanidad animal, $T$. gondii, constituye una de las principales causas de problemas reproductivos en pequeños rumiantes a nivel mundial, especialmente en cabras (Dubey et al., 2020). Provoca reabsorción fetal, abortos, mortinatos y mortalidad neonatal que resultan en grandes pérdidas económicas (Freyre et al., 1999; Dubey et al., 2020).

Existen numerosos estudios de la seroprevalencia de $T$. gondii en rumiantes menores, Así, se reportan prevalencias altas en ovejas y cabras en las islas caribeñas de Dominica (67 y 58\%), Granada (48 y 57\%), Montserrat (89 y $80 \%$ ) y St. Kitts y Nevis (57 y $42 \%$ ), respectivamente (Hamilton et al., 2014), en tanto que seroprevalencias bajas (15.8 y $3.8 \%$ ) en cabras y ovejas de Pernambuco y Paraíba al noreste de Brasil, respectivamente (da Silva et al., 2015). La prevalencia de toxoplasmosis varía con la ubicación geográfica, sistema de crianza y con la edad del animal (De Moura et al., 2016, Rêgo et al., 2016; Tegegne et al 2016). Por otro lado, estudios sobre prevalencia de $T$. gondii en pequeños rumiantes en Perú son escasos; así Bernal et al. (2015) y Caldas et al. (2007) hallaron prevalencias de 44 y $65.7 \%$ en ovinos de Puno y Junín.

La crianza de cabras en el país es una actividad secundaria, pero representa un medio de subsistencia importante para la población rural (Arroyo, 2007). La crianza de caprinos se lleva a cabo principalmente en la costa y sierra del país, encontrándose en mínima cantidad en la zona de selva (GómezUrviola et al., 2016). En la costa, los departamentos con mayor producción son Piura, Lima e Ica y en la sierra Ayacucho y Huancavelica (MINAGRI, 2015). Su crianza está mayormente orientada a la producción de carne de cabrito y leche para la producción de queso (Minagri, 2015). Por lo tanto, el objetivo del estudio fue determinar la seroprevalencia de Toxoplasma gondii y su asociación con los factores de riesgo en la infección de caprinos en el país.

\section{Materiales y Métodos}

\section{Animales y Lugar de Estudio}

La población caprina considerada en el estudio provino de 23 de los 24 departamentos que conforman el país. La población caprina de Ancash no fue considerada debido a problemas climatológicos (fenómeno del niño costero) que imposibilitaron la toma de muestras. El grupo racial predominantes es el criollo, la crianza es mayormente de tipo extensiva y la alimentación se basa principalmente en pastos naturales y subproductos y rastrojos de la agricultura. Cómo criterio de inclusión; se consideró animales aparentemente saludables mayores de seis meses, excluyéndose a los animales con menos de 30 días en las zonas de muestreo.

La zonas costa norte y centro-sur presenta altitudes entre 0 y $2000 \mathrm{~m}$, temperatura ambiental de $18-27^{\circ} \mathrm{C}$. y precipitación anual promedio de 0 y $500 \mathrm{~mm}$, respectivamente. La zona de la sierra presenta altitudes entre 2000 y 6750 m, pero las cabras se encuentran por debajo de los $4000 \mathrm{~m}$, donde la temperatura oscila entre $10 \mathrm{y} 15^{\circ} \mathrm{C}$ y la precipitación pluvial entre 500 y 700 mm/año. La zona de selva (oriente) presenta altitudes bajas (80-500 msnm) y altas (500-2000 msnm), temperaturas de $22-26^{\circ} \mathrm{C}$ y precipitación entre 500 a 5000 mm/año (Clima del Perú, s.f.)

\section{Muestras}

Las muestras de sangre fueron colectadas en forma aleatoria simple de cabras por el Servicio Nacional de Sanidad Animal (SENASA) durante los años 2017 y 2018 para el monitoreo sanitario de Brucella melitensis. Las muestras de suero sanguíneo $(n=6625)$ fueron conservadas en conge- 
lación $\left(-20^{\circ} \mathrm{C}\right)$, que luego de ser trabajadas para su propósito original, fueron donadas a la Facultad de Medicina Veterinaria de la Universidad Nacional Mayor de San Marcos para el estudio de otras enfermedades de importancia sanitaria.

Los sueros de los animales fueron agrupados en tres zonas de procedencia de acuerdo con los departamentos con mayor producción caprina (Sarria et al., 2014), pero en la zona de Sierra se incorporó a 15 animales procedentes de tres departamentos de la selva.

- Zona Costa Norte: Tumbes, Piura, Lambayeque

- Zona Costa Centro-Sur: La Libertad, Lima, Ica, Arequipa, Moquegua, Tacna

- Zona Sierra-Oriente: Cajamarca, Amazonas, Huánuco, San Martín, Pasco, Junín, Huancavelica, Ayacucho, Apurímac, Cusco, Puno por parte de los departamentos de la sierra, incluyendo además los departamentos de la zona oriental como Loreto, Ucayali y Madre de Dios

Las muestras se clasificaron según la edad de las cabras ( $<1,1-3,>3$ años), sexo, procedencia (Zona Costa Norte, Zona Costa Centro-Sur, Zona Sierra-oriente, altitud (0$500,>500-2500,>2500 \mathrm{msnm}$ ) y tipo de crianza (intensiva, extensiva). El estudio no afectó a los animales pues se hizo a partir de muestras de un Banco de Sueros.

\section{Tamaño de Muestra}

Se tomó de referencia la población caprina del IV Censo Nacional Agropecuario 2012 (INEI, 2012), sin incluir los animales de Ancash, la prevalencia $(23.7 \%)$ reportada por Dong et al. (2018) en un estudio similar, un nivel de confianza del $95 \%$ y un error máximo admisible del $5 \%$. Se aplicó la fórmula de tamaño mínimo de muestra para proporciones en poblaciones finitas (Daniel, 2002). El tamaño mínimo muestral resultante fue de 278 animales; sin embargo, debido a la existencia de las muestras de suero, se cuadruplicó el tamaño muestral de cada departamento, trabajándose finalmente con 1119 individuos.

\section{Análisis de Laboraorio}

Se determinó la presencia de anticuerpos contra Toxoplasma gondii como variable dependiente. Se utilizó un kit comercial de ELISA indirecta (ID Screen Toxoplasmosis Indirect Multi species, ID.Vet, Francia). Se siguió el protocolo del fabricante. Se determinó la densidad óptica (DO) de las muestras y de los controles negativo y positivo. Se utilizó la fórmula $\mathrm{S} / \mathrm{P} \%=(\mathrm{DO}$ media de la muestra - DO media del control negativo) / (DO media del control positivo - DO media del control negativo) $* 100$. Los resultados se consideraron como negativo ( $\mathrm{S} / \mathrm{P} \%$ $\leq 40 \%)$, dudoso $(>40-<50 \%)$ y positivo $(\geq 50 \%)$. La lectura de la absorbancia se realizó en el lector de ELISA Kayto-RT-2100C con filtro de $450 \mathrm{~nm}$.

\section{Análisis de la Información}

La prevalencia de anticuerpos contra $T$. gondii se expresa en forma porcentual con sus respectivos intervalos de confianza al $95 \%$. En el análisis bivariado se utilizaron las pruebas de Chi cuadrado y regresión logística para determinar el grado de asociación entre la presencia de anticuerpos contra el parásito con las variables en estudio. En el análisis multivariado se utilizó la regresión logística múltiple para obtener los valores del Odds ratio (OR) a fin de estimar los factores de riesgo $(\mathrm{p}<0.05)$.

\section{Resultados}

La prevalencia general de $T$. gondii en caprinos distribuida por los 23 departamentos evaluados fue de $28.15 \%$ (315/1119) (Cuadro 1). Cinco muestras mostraron resultados dudosos, por lo que no se incluyeron en las demás evaluaciones del estudio. 
Cuadro 1. Prevalencia general (\%) de Toxoplasma gondii en caprinos de 23 departamentos del Perú (2017-2018)

\begin{tabular}{lcccc}
\hline & & \multicolumn{3}{c}{ Muestras } \\
\cline { 3 - 5 } Departamento & $\mathrm{n}$ & $\begin{array}{c}\text { Positivas } \\
(\%)\end{array}$ & $\begin{array}{c}\text { Negativas } \\
(\%)\end{array}$ & $\begin{array}{c}\text { Dudosas } \\
(\%)\end{array}$ \\
\hline Amazonas & 11 & 36.36 & 63.64 & 0 \\
Apurímac & 42 & 16.67 & 83.33 & 0 \\
Arequipa & 47 & 17.02 & 82.98 & 0 \\
Ayacucho & 120 & 27.50 & 71.67 & 0.83 \\
Cajamarca & 77 & 19.48 & 79.22 & 1.30 \\
Cusco & 53 & 18.87 & 81.13 & 0 \\
Huancavelica & 74 & 36.49 & 63.51 & 0 \\
Huánuco & 47 & 21.28 & 78.72 & 0 \\
Ica & 78 & 48.72 & 51.28 & 0 \\
Junín & 27 & 14.81 & 85.19 & 0 \\
La libertad & 63 & 42.86 & 55.56 & 1.59 \\
Lambayeque & 54 & 38.89 & 61.11 & 0 \\
Lima & 51 & 25.49 & 74.51 & 0 \\
Loreto & 5 & 100 & 0 & 0 \\
Madre de Dios & 5 & 40 & 60 & 0 \\
Moquegua & 13 & 30.77 & 69.23 & 0 \\
Pasco & 9 & 0 & 100 & 0 \\
Piura & 242 & 27.27 & 71.90 & 0.83 \\
Puno & 5 & 0 & 100 & 0 \\
San Martín & 3 & 100 & 0 & 0 \\
Tacna & 21 & 14.29 & 85.71 & 0 \\
Tumbes & 17.91 & 82.09 & 0 \\
Ucayali & 67 & 60.00 & 40 & 0 \\
\hline Total (\%) & 5 & 315 & 71.40 & 5 \\
Total (animales) & 119 & & 799 & \\
\hline & & & & 0.45 \\
\hline
\end{tabular}

No hubo asociación significativa ( $\mathrm{p}>0.05$ ) entre la prevalencia de T. gondii con la edad ni con el sexo de los animales (Cuadro 2). Por otra parte, Se encontró una asociación significativa $(\mathrm{p}<0.05)$ entre la prevalencia de Toxoplasma gondii con las variables localización $(\mathrm{p}=0.038)$ siendo mayor en la zona costa centro sur (34.19\%), altitud $(\mathrm{p}=0.001)$ siendo mayor zonas entre 0 y 500 msnm (32.68\%), con el tipo de crianza $(\mathrm{p}=0.026)$, siendo mayor en crianza de tipo intensiva (34.42\%) (Cuadro 2).

El análisis de regresión logística múltiple, ajustado mediante Stepwise, indicó asociación significativa $(\mathrm{p}<0.05)$ con las variables localización, altitud y sistema de crianza (Cuadro 3). 
Cuadro 2. Análisis univariado de los factores asociados al diagnóstico serológico de Toxoplasma gondii en caprinos del Perú (2017-2018)

\begin{tabular}{|c|c|c|c|}
\hline \multirow{2}{*}{ Variables } & \multicolumn{2}{|c|}{$\begin{array}{c}\text { Diagnóstico serológico de toxoplasmosis } \\
\text { caprina }\end{array}$} & \multirow{2}{*}{$p^{*}$} \\
\hline & $\begin{array}{c}\text { Positivos }(\mathrm{n}=315) \\
\mathrm{n}(\%)\end{array}$ & $\begin{array}{c}\text { Negativos }(\mathrm{n}=799) \\
\mathrm{n}(\%)\end{array}$ & \\
\hline Edad (años) & & & 0.433 \\
\hline$<1$ & $20(24.69)$ & $61(75.31)$ & \\
\hline $1-3$ & $254(28.00)$ & $653(72.00)$ & \\
\hline$>3$ & $41(32.54)$ & $85(67.46)$ & \\
\hline Sexo & & & 0.697 \\
\hline Hembra & $308(28.36)$ & $778(71.64)$ & \\
\hline Macho & $7(25)$ & $21(75)$ & \\
\hline Localización & & & 0.038 \\
\hline Zona Costa Norte & $99(27.42)$ & $262(72.58)$ & \\
\hline Zona Costa Centro-Sur & $93(34.19)$ & $179(65.81)$ & \\
\hline Zona Sierra-Oriente & $123(25.57)$ & $358(74.43)$ & \\
\hline Altitud (msnm) & & & 0.001 \\
\hline $0-500$ & $149(32.68)$ & $307(67.32)$ & \\
\hline$>500-2500$ & $63(31.66)$ & $136(68.34)$ & \\
\hline$>2500$ & $103(22.44)$ & $356(77.56)$ & \\
\hline Tipo de crianza & & & 0.026 \\
\hline Extensivo & $241(26.81)$ & $658(73.19)$ & \\
\hline Intensivo & $74(34.42)$ & $141(65.58)$ & \\
\hline
\end{tabular}

Los caprinos de la Zona Sierra-Oriente y de la Zona Costa Centro-Sur presentaron un riesgo (OR) de 1.62 y 1.67 veces más de presentar anticuerpos contra $T$. gondii, respectivamente que aquellos de la Zona Costa Norte, considerando que la altitud y el sistema de crianza permanecen constantes. Los caprinos muestreados a altitudes de $0-500 \mathrm{y}$ $>500-2500$ msnm presentaron 2.59 y 2.02 veces más de riesgo (OR) de presentar anticuerpos contra el parásito que aquellos ubicados a una altitud $>2500 \mathrm{msnm}$, considerando que la localización y el sistema de crianza permanecen constantes. Asimismo, aque- llos criados bajo un sistema intensivo presentaron un mayor riego $(\mathrm{OR}=1.58)$ que aquellos criados en sistema extensivo (Cuadro 3 ).

\section{Discusión}

La prevalencia de Toxoplasma gondii en caprinos mediante la técnica de ELISA indirecta fue del $28.15 \%$. Los resultados, además, indicaron que en 21 de los 23 departamentos en estudio se encontró animales serorreactores a $T$. gondii, con prevalencias entre 14.29 hasta $100 \%$. Solo los departa- 
Cuadro 3. Análisis multivariado de factores asociados al diagnóstico serológico de Toxoplasma gondii en caprinos mediante regresión logística múltiple

\begin{tabular}{|c|c|c|c|c|}
\hline \multirow{2}{*}{$\begin{array}{l}\text { Variables } \\
\text { Localización }\end{array}$} & \multirow[t]{2}{*}{ Odds Ratio } & \multicolumn{2}{|c|}{$\begin{array}{c}\text { Intervalo de confianza } \\
\text { al } 95 \%\end{array}$} & \multirow[t]{2}{*}{ Valor $\mathrm{p}$} \\
\hline & & & & \\
\hline Sierra-Oriente & 1.62 & 1.00 & 2.61 & 0.049 \\
\hline Costa centro-sur & 1.67 & 1.12 & 2.48 & 0.011 \\
\hline Costa norte & 1 & & & \\
\hline \multicolumn{5}{|l|}{ Altitud } \\
\hline $0-500 \mathrm{msnm}$ & 2.59 & 1.67 & 4.02 & $<0.001$ \\
\hline$>500-2500 \mathrm{msnm}$ & 2.02 & 1.34 & 3.05 & 0.001 \\
\hline$>2500 \mathrm{msnm}$ & 1 & & & \\
\hline \multicolumn{5}{|l|}{ Sistema de crianza } \\
\hline Intensivo & 1.58 & 1.11 & 2.27 & 0.012 \\
\hline Extensivo & 1 & & & \\
\hline
\end{tabular}

mentos de Pasco y Punto estuvieron libres de caprinos seropositivos a $T$. gondi, pero es posible que se haya debido al reducido número de muestras en dichas localidades.

El $28.15 \%$ de prevalencia fue inferior al $35.6 \%$ reportado por Rivera et al. (1988) en seis departamentos del Perú mediante la técnica de HAI. Sin embargo, Dubey et al. (1995), señala que esta prueba presenta baja sensibilidad (29.4\%) y especificidad (98.3\%), en tanto que la prueba de ELISA empleada en el presente estudio tiene una sensibilidad del $98.1 \%$ y una especificidad del $99.8 \%$ según el laboraorio fabricante del kit (Mangili et al., 2009). Por otro lado, no es dable comparar la seroprevalencia promedio del estudio $(28.15 \%)$ con los valores hallados entre 0 y $100 \%$ de otras ciudades o regiones, ya que este valor promedio representa el efecto de varias variables, los cuales deben ser agrupadas según los factores de riesgo reportado en la literatura, para llegar a conclusiones generales y comparables (Stelzer et al., 2019). De otra parte, los resultados dudosos encon- trados $(\mathrm{n}=5)$ con la técnica de ELISA podrían indicar que aquellos animales se hallarían con una infección aguda por $T$. gondii, donde la IgM se encontraría más elevada que la IgG, único anticuerpo que es detectado por la prueba de ELISA utilizada (Lopes et al., 2013).

La prueba de Chi cuadrado indicó que solo las variables localización, altitud y tipo de crianza; presentaron asociación significativa con la presencia de anticuerpos contra $T$. gondii, a pesar de otros estudios que evidencian una seropositividad proporcional a la edad del animal (Anderlini et al., 2011; Garcia et al., 2012; Xu et al., 2015; Rêgo et al., 2016). Este resultado podría deberse que solo $7.24 \%$ de los animales pertenecían al grupo menor de un año y probablemente estos eran más cercanos al año de edad, dado que los ganaderos evitan tomar muestras en cabritos de corta edad. Esto indicaría que los animales de menor edad del estudio habrían tenido tiempo suficiente para estar expuestos a los ooquistes presentes en el medio ambiente. 
La variable sexo no constituyó factor de riesgo, mientras que otros autores hallaron un mayor riesgo de infección en hembras (Ahmad et al., 2015; de Moura et al., 2016; Rêgo et al., 2016), debido a una mayor susceptibilidad a la infección por parásitos protozoarios en las hembras en comparación con los machos (Alexander y Stinson, 1988), además que otros factores como preñez, nutrición y lactación (Martin, 2000; Kelly et al., 2001).

El análisis multivariado evidenció que las variables localización, altitud y sistema de crianza constituyeron factores de riesgo para la presentación de la infección por $T$ gondii. Respecto a la «localización», si bien los animales criados en la zona sierra-oriente mostraron un $\mathrm{OR}=1.62$, es posible que el resultado haya estado influenciado por la inclusión de 15 animales criados en la zona oriente que mostraron prevalencias entre 40 y $100 \%$. Por otro lado, la mayor prevalencia de los caprinos de la Zona Costa Centro-Sur (34.19\%) no solo se deba a la zona geográfica sino a otras no contempladas en el estudio, como fuentes de agua, cercanía a zonas urbanas, presencia de gatos, etc., que estarían afectando la presencia del parásito. Otros estudios también han encontrado diferencias significativas entre zonas geográficas (Tzanidakis et al., 2012; Alvarado-Esquivel et al., 2013; de Moura et al., 2016; Zou et al., 2015), aunque $\mathrm{Xu}$ et al. (2015) y Tilahun et al. (2018) no encontraron tales diferencias.

En el presente estudio se evidenció un mayor riesgo (OR) de presentar anticuerpos contra $T$. gondii en caprinos criados entre 0 y $2500 \mathrm{msnm}$ en comparación con caprinos criados en altitudes mayores a $2500 \mathrm{msnm}$, a diferencia de otros reportes que indican un mayor riesgo en crianzas en zonas montañosas (Alvarado-Esquivel et al., 2013; De Moura et al., 2016; Tegegne et al., 2016). Estas diferencias con las del presente estudio podrían deberse a otros factores no considerados como la cercanía a zonas urbanas donde la presencia de gatos es más evidente
(García et al., 2012; Ahmad et al 2015; de Moura et al., 2016), y la presencia de fuentes de agua.

Mientras que los caprinos criados bajo un sistema intensivo, el riesgo (OR) de presentar anticuerpos contra $T$. gondii fue de 1.58 veces el de los caprinos criados en un sistema extensivo, siempre y cuando la localización y la altura fueran similares. Todos estos resultados evidenciarían falta de conocimiento sobre las medidas de un manejo técnico y de asesoramiento de algunos productores en este tipo de crianza.

Los sistemas de producción están relacionados con acciones específicas bajo las cuales los animales son criados y alimentados. Estas acciones, podrían influir en la probabilidad de infección con ooquistes de $T$. gondii, presentes en el agua o en alimentos contaminados. Se espera que la crianza intensiva bien llevada disminuye el riesgo de infección con T. gondii (Xu et al., 2015; Rêgo et al., 2016) al evitar la presencia de hospederos definitivos del parásito y otras medidas sanitarias y de bioseguridad (Luyo et al., 2017); sin embargo, el concentrado utilizado para alimentar a las cabras, especialmente en las ganaderías lecheras favorecería la presencia de roedores, estando los productores propensos a tener gatos como medida «protectiva», favoreciendo de esta manera la presencia continua de ooquistes de $T$. gondii en el ambiente (Gebremedhin et al., 2013).

\section{Conclusiones}

- Se confirma que T. gondii es una infección endémica en caprinos criados en casi la totalidad de departamentos del país.

- La prevalencia en las diversas regiones estaría influenciada por factores de riesgo relacionados con la localización (sierraoriente y costa centro-sur), altitud ( $<2500$ msnm) y tipo de crianza (intensiva). 


\section{Literatura Citada}

1. Ahmad N, Iqbal Z, Mukhtar M, Mushtaq M, Khan KM, Qayyum M. 2015. Seroprevalence and associated risk factors of toxoplasmosis in sheep and goats in Pothwar Region, Northern Punjab, Pakistan. Pakistan J Zool 47: 161-167.

2. Alexander J, Stinson WH. 1988. Sex hormones and the course of parasitic infection. Parasitol Today 4: 189-193. doi: 10.1016/0169-4758(88)90077-4

3. Alvarado-Esquivel C, Silva-Aguilar D, Villena I, Dubey JP. 2013. Seroprevalence of Toxoplasma gondii infection in dairy goats in Michoacan State, Mexico. J Parasitol 99: 540-542. doi: 10.1645/12-103.1

4. Anderlini GA, Mota RA, Faria EB, Cavalcanti EFTSF, Valença RMB, Pinheiro Júnior JW, de Albuquerque PPF, Neto OLS. 2011. Occurrence and risk factors associated with infection by Toxoplasma gondii in goats in the state of Alagoas, Brazil. Rev Soc Bras Med Trop 44: 157-162. doi: 10.1590/s003786822011005000017

5. Arroyo O. 2007. Situacion actual y proyecciones de la crianza de caprinos en el Perú. Arch Latinoam Prod Anim 15(Supl. 1): 163-168.

6. Bernal D, Suárez F, Huanca W, Chávez A. 2015. Prevalencia de toxoplasmosis ovina en dos localidades de Puno, Perú. Rev Inv Vet Perú 26: $291-$ 295. doi: 10.15381/rivep.v26i2.11002

7. Caldas JP, Chávez VA, Casas AE. 2006. Seroprevalencia del Toxoplasma gondii en borregas de una empresa ganadera de la Sierra Central. Rev Inv Vet Perú 17: 14-19.

8. Clima del Perú. s.f. En: Wikipedia. [Internet]. Disponible en: https:// es.wikipedia.org/wiki/Clima_del Per\%C3\%BA\#Referencias

9. Cook AJC, Gilbert RE, Buffolano W, Zufferey J, Eskild P, Jenum PA, Foulon W, et al. 2000. Sources of
Toxoplasma infection in pregnant women: European multicentre case-control study. BMJ Clin Res 321(7254): 142147. doi: $10.1136 / \mathrm{bmj} .321 .7254 .142$

10. da Silva JG, Alves, BHLS, Melo RPB, Kim PCP, Neto OLS, Bezerra MJG, et al. 2015. Occurrence of anti-Toxoplasma gondii antibodies and parasite DNA in raw milk of sheep and goats of local breeds reared in Northeastern Brazil. Acta Trop 142: 145-148. doi: 10.1016/ j.actatropica.2014.11.011

11. Daniel W. 2002. Bioestadística. Base para el análisis de las ciencias de la salud. México: México: Limusa. 755 p.

12. De Moura AB, Ribeiro A, De Souza AP, Da Silva MI, Machado G, Klauck V, Pazinato R. Da Silva AS. 2016. Seroprevalence and risk factors for Toxoplasma gondii infection in goats in southern Brazil. Acta Scie Vet 44: 1367.

13. Dubey JP, Thulliez P, Weigel RM, Andrews CD, Lind P, Powell EC. 1995. Sensitivity and specificity of various serologic tests for detection of Toxoplasma gondii infection in naturally infected sows. Am J Vet Res 56: 1030-1036.

14. Dubey JP, Jones JL. 2008. Toxoplasma gondii infection in humans and animals in the United States. Int $\mathbf{J}$ Parasitol. 38: 1257-1278. doi: 10.1016/ j.ijpara.2008.03.007

15. Dubey JP, Murata FHA, CerqueiraCézar CK, Kwok OCH. 2020. Public health and economic importance of Toxoplasma gondii infections in goats: the last decade. Res Vet Sci. 132: 292307. doi: $10.1016 /$ j.rvsc. 2020.06 .014

16. Dong H, Su R, Lu Y, Wang M, Liu J, Jian F. Yang Y. 2018. Prevalence, risk factors, and genotypes of Toxoplasma gondii in food animals and humans (2000-2017) from China. Front Microbiol 9. doi: 10.3389/fmicb.2018.02108

17. Freyre A, Bonino J, Falcon J, Castells D, Correa O, Casaretto A. 1997. The incidence and economic significance of ovine toxoplasmosis in Uruguay. Vet Parasitol 73: 13-15. doi: 10.1016/s03044017(97)00069-1 
18. FAO-WHO. 2015. Joint $\mathrm{FAO} / \mathrm{WHO}$ food standards pro-gram codex committee on food hygiene. Forty-seventh session. Boston, Massachusetts, USA.

19. Garcia G, Sotomaior C, do Nascimento AJ, Navarro IT, Soccol VT. 2012. Toxoplasma gondii in goats from Curitiba, Paraná, Brazil: risks factors and epidemiology. Rev Bras Parasitol Vet 21: 42-47. doi: /10.1590/S198429612012000100009

20. Gebremedhin EZ, Agonafir A, Tessema TS, Tilahun G, Medhin G, Vitale M, DiMarco V, et al. 2013. Seroepidemiological study of ovine toxoplasmosis in East and West Shewa Zones of Oromia Regional State, Central Ethiopia. BMC Vet Res 9: 117. doi: 10.1186/17466148-9-117

21. Gómez-Urviola NC, Gómez-Urviola $J W$, Celi-Mariátegui IDR, MilánSendra MJ, Jordana-Vidal J. 2016. La cabra criolla peruana, situación actual y perspectivas conservacionistas. En: Vargas JE, Zaragoza LM, Delgado JV, Rodríguez GG (eds). Biodiversidad caprina iberoamericana. Bogot[a, Colombia: Univ. Cooperativa de Colombia. p 163-168.

22. Hamilton CM, Katzer F, Innes EA. Kelly PJ. 2014. Seroprevalence of Toxoplasma gondii in small ruminants from four Caribbean islands. Parasit Vectors 7:449. doi: 10.1186/1756-3305-7-449

23. [INEI] Instituto Nacional de Estadística e Informática. 2012. IV Censo Nacional Agropecuario 2012. [Internet] Disponible en: http://censos.inei.gob.pe/ cenagro/tabulados/?id=Censos Nacionales

24. Kelly A, Messingham N, Elizabeth AH Kovacs J. 2001. Estrogen restores cellular immunity in injured male mice via suppression of interleukin- 6 production. J Leuk Biol 70: 887-895.

25. Lopes AP, Dubey JP, Neto F, Rodrigues A, Martins T, Rodrigues M, Cardoso L. 2013. Seroprevalence of Toxoplasma gondii infection in cattle, sheep, goats and pigs from the north of
Portugal for human consumption. Vet Parasitol 193: 266-269. doi: 10.1016/ j.vetpar.2012.12.001

26. Luyo C. Pinedo R, Chávez A, Casas E. 2017. Factores asociados a la seroprevalencia de Toxoplasma gondii en cerdos de granjas tecnificadas y no tecnificadas de Lima, Perú. Rev Inv Vet Perú 28: 141-149. doi: 10.15381/rivep.v28i1.12930

27. Martin JT. 2000. Sexual dimorphism in immune function: the role of prenatal exposure to androgens and estrogens. Eur J Pharmacol 405: 251-261. doi: 10.1016/S0014-2999(00)00557-4

28. Mangili PM, Vesco G, Feliziani F, Paoloni A, Menichelli M, Cagiola M, Marini C, et al. 2009. Development and evaluation of the performance of an in-house ELISA to be used for the indirect diagnosis of toxoplasmosis in sheep. In: Meeting of the Società Italiana di Diagnostica di Laboratorio Veterinaria - SIDILV. Parma, Italy.

29. [MINAGRI] Ministerio de Agricultura y Riego. 2015. Situación de las actividades de crianza y producción de caprinos. [Internet]. Disponible en: http:// minagri.gob.pe/portal/40-sector-agrario/ situacion-de-las-actividades-de-crianzay-producci/299-caprinos?limitstart $=0$

30. Montoya JG, Liesenfeld O. 2004. Toxoplasmosis. Lancet 363: 1965-1976. doi: 10.1016/S0140-6736(04)16412-X

31. Rani S, Cerqueira-Cézar CK, Murata FHA, Kwok OCH, Dubey JP, Pradhan A. 2020. Distribution of Toxoplasma gondii tissue cysts in shoulder muscles of naturally infected goats and lambs. J Food Prot 83: 13961401. doi: 10.4315/JFP-20-024

32. Rêgo WMF, Paula NRO, Vitor RWA, Silva RAB, Diniz BLM, Sousa MM, Coelho WAC, et al. 2016. Risk factors for Toxoplasma gondii infection in goats and sheep raised in the State of Piaui in northeast Brazil. Small Ruminant Res 141: 17-23. doi: 10.1016/j.smallrumres.2016.04.010 
33. Rivera H, Ameghino E, Samamé H, Lévano J. 1988. Estudio de la toxoplasmosis en caprinos. En:XI Reunión Científica Anual de la Asociación Peruana de Producción Animal. Piura, Perú.

34. Sarria J, Ruiz F, Mena Y, Castel J. 2014. Caracterización y propuestas de mejora de los sistemas de producción caprina de la costa central del Perú. Rev Mex Cienc Pec 5: 409-427.

35. Scallan E, Hoekstra RM, Angulo FJ, Tauxe RV, Widdowson M, Roy SL, et al. 2011. Foodborne illness acquired in the United States - major pathogens. Emerg Infec Dis 17: 7-15. doi: 10.3201/ eid1701.P11101

36. Stelzer S, Basso W, Benavides Silván J, Ortega-Mora LM, Maksimov P, Gethmann J, Conraths FJ, Schares G. Toxoplasma gondii infection and toxoplasmosis in farm animals: risk factors and economic impact. Food Waterborne Parasitol 15: e00037. doi: 10.1016/j.fawpar.2019.e00037

37. Tenter AM, Heckeroth AR, Weiss LM 2000. Toxoplasma gondii: from animals to humans. Int J Parasitol 30: 1217-1258.

38. Tegegne D, Kelifa A, Abdurahaman M, Yohannes M. 2016. Seroepidemiology and associated risk factors of
Toxoplasma gondii in sheep and goats in Southwestern Ethiopia. BMC Vet Res 12: 280. doi: 10.1186/s12917-016-0906-2

39. Tilahun B, Tolossa YH, Tilahun G, Ashenafi H, Shimelis S. 2018. Seroprevalence and risk factors of Toxoplasma gondii infection among domestic ruminants in East Hararghe zone of Oromia region, Ethiopia. Vet Med Int 2018: ID 4263470. doi: 10.1155/2018/ 4263470

40. Tzanidakis N, Maksimov P, Conrath, FJ, Kiossis E, Brozos C, Sotiraki S, Schares G. 2012. Toxoplasma gondii in sheep and goats: seroprevalence and potential risk factors under dairy husbandry practices. Vet Parasitol 190: 340-348. doi: 10.1016/j.vetpar.2012.07.020

41. Xu P, Li X, Tang F, Liu YH, Kou X, Zhao ML, Li B, et al. 2015. Seroprevalence and risk factors for Toxoplasma gondii in sheep and goats in Jinzhou, Northeastern China. Trop Biomed 32: 563-567.

42. Zou F, Yu X, Yang Y, Hu S, Chang H, Yang J, Duan G 2015. Seroprevalence and risk factors of Toxoplasma gondii infection in buffaloes, sheep and goats in Yunnan Province, Southwestern China. Iran J Parasitol 10: 648-651. 